\title{
Secondary Central Nervous System Lymphoma presenting as Fever of Unknown Origin
}

Sean Patrick Bliven ( $\nabla$ pbliven@uabmc.edu )

University of Alabama at Birmingham

Mayur Narkhede

University of Alabama at Birmingham

\section{Case Report}

Keywords: Diffuse large B-cell lymphoma, Secondary central nervous system lymphoma, Fever of unknown origin

Posted Date: February 22nd, 2022

DOI: https://doi.org/10.21203/rs.3.rs-1374908/v1

License: (c) (i) This work is licensed under a Creative Commons Attribution 4.0 International License. Read Full License 


\section{Abstract}

Background: Diffuse large B-cell lymphoma is a form of non-Hodgkin's lymphoma that routinely presents with systemic "B" symptoms including fever of unknown origin. After systemic treatment, patients can relapse with secondary central nervous system lymphoma. These patients typically present with neurological symptoms and not systemic "B" symptoms.

Case presentation: We describe the case of a woman in her fifties that presented with fever of unknown origin as her initial presenting symptom of relapsed isolated secondary central nervous system lymphoma. MR brain showed parenchymal lymphomatous involvement compatible with CNS lymphoma. Her fevers resolved after central nervous system targeted chemotherapy with associated improvement on imaging.

Conclusion: Isolated central nervous system lymphoma (primary or secondary) can lead to fever of unknown origin. We recommend evaluation for CNS recurrence among patients with lymphoma that present with fever of unknown origin.

\section{Background}

Diffuse large B-cell lymphoma ( $\mathrm{DLBCL}$ ) is a relatively common type of non-Hodgkin's lymphoma. Median age at diagnosis is 64 years, with most patients presenting with lymphadenopathy, up to forty percent of patients with extranodal disease, and greater than sixty percent with advanced stage disease. [citation 1 and 2]. Patients' symptoms at diagnosis vary based on the organ affected, although up to 30 percent of patients with DLBCL can present with "B" symptoms, including fever, weight loss, and night sweats. [citation 3].

A feared complication of DLBCL is CNS recurrence after systemic treatment. Up to $40 \%$ of patients have some form of recurrence. [citation 12] CNS relapse rates are low, with studies estimating rates of two to six percent among patients with a form of aggressive lymphoma (including DLBCL). [citations 4, 13]. Symptoms of CNS recurrence are similar to those of isolated primary CNS lymphoma (CNS lymphoma without a concurrent systemic lymphoma, PCNSL) and include headaches, focal neurological deficits, lumbosacral pain, mental status changes, and even seizures [citation 5]. Fever and other systemic "B" symptoms are notably absent in isolated CNS lymphoma.

Fever of unknown origin (FUO) is defined as a temperature greater than $38.3^{\circ} \mathrm{C}$ on several occasions, lasting at least three weeks, and with an uncertain diagnosis after one week in the hospital. [citation 11]. Today, workup includes physical examination, cultures, and imaging and an empiric course of antibiotics. Malignancy associated fever of unknown origin is a diagnosis of exclusion. Castleman's disease, lymphoma, hemophagocytic lymphohistiocytosis, and renal cell carcinoma remain common causes of malignancy associated with FUO.

\section{Case Presentation}


The patient is a woman in her 50 s that initially presented with left-sided sciatic pain. She was initially treated conservatively with physical therapy and sciatic nerve injections. After 10 months her symptoms did not resolve and further imaging of the lumbar spine and left leg revealed a large left leg mass. Pathology revealing non-GCB, double expresser (c-Myc greater than 70\% and BCL-2 greater than 60\%) diffuse large B-cell lymphoma (DLBCL). Initial PET scan showed an additional lesion in the retroperitoneum but no disease above the diaphragm (stage IV disease). At the time of diagnosis she had no further past medical history, surgical history notable for only an appendectomy, and no relevant family history.

She was started on R-CHOP like induction chemotherapy as part of a clinical trial. Her induction treatment was complicated by COVID-19 infection, for which she received bamlanivimab. Her end of treatment PET scan showed a complete metabolic response.

Four months after complete response she began having persistent fevers. Her temperature varied from 100.8 to 104 with the highest fevers typically occurring in the evening. She was not neutropenic and extensive culture data including blood, urine, and CSF were all negative, as well as HSV, CMV, and VZV PCR negative of both the serum and CSF. Her fevers continued for over a month. She had an episode of headache and fever prompting CNS workup. MRI brain showed diffuse parenchymal lymphomatosis involvement including the basal ganglia, splenium of the corpus callosum, and cerebral peduncle concerning for CNS lymphoma. CT of the chest, abdomen, and pelvis and MRI of the femur showed no evidence of non-CNS recurrence. Given the extensive negative infectious workup, her recurrent fevers were determined to be secondary to CNS lymphoma and she was started on further chemotherapy with MATRIX.

Her fevers resolved after initiation of CNS targeted chemotherapy. Interim brain MR after three cycles of MATRIX showed interval decrease in size of the lesions in her basal ganglia, corpus callosum, and cerebral peduncle.

\section{Discussion And Conclusions}

Our case report is unique because it describes a novel case of fever of unknown origin secondary to isolated secondary CNS lymphoma without an associated systemic lymphoma relapse.

Review of the literature reveals only two similar cases. The first case, described in 2001, was in a patient that presented with pituitary lymphoma and fever was thought to be a localized effect of the lymphoma, in this case secondary to extrasellar leakage of blood. [citation 8]. The second case, described in 2009 by Salih, Saeed, et al, described a patient that presented with intraparenchymal primary CNS lymphoma without other cause. [citation 9].

The mechanism of fever among patients with systemic lymphoma is not entirely understood, but is believed to be secondary to elevated levels of IL- 6 and IL-10, with higher levels of IL- 6 among patients with B-symptoms. [citation 7] Elevated serum levels of IL-6 and IL-10 have not been reported in the 
literature among patients with isolated PCNSL. Instead, the elevated levels of IL-6 and IL-10 are found in higher concentration in CSF. [citation 10] We theorize that, in the majority of cases of CNS lymphoma, the localization of the inflammatory interleukins to CSF may preclude the same systemic inflammation leading to fevers in patients with PCNSL or isolated SCNSL compared to those with systemic DLBCL.

However, for reasons not yet understood, our case and that reported by Salih, Saeed, et al, describe cases in which the patient's localized CNS lymphoma led to activation of the inflammatory cascade and Bsymptoms typically only seen in systemic lymphoma. Based on our experiences, CNS lymphoma remains a possible diagnosis for patients that present with FUO. For all comers that present with FUO, we recommend typical work up for infectious sources. However, in patients with a history of DLBCL that present with persistent fever of unknown origin, we recommend evaluation for CNS recurrence in addition to evaluation for systemic recurrence and infectious sources.

\section{Abbreviations}

DLBCL

Diffuse large B-cell lymphoma

FUO

Fever of unknown origin

PCNSL

Primary CNS lymphoma

SCNSL

Secondary CNS lymphoma

\section{Declarations}

- Ethics approval and consent to participate

- The patient was cared for at the University of Alabama Birmingham Hospital. UAB's Institutional Review Board does not require review for publication of a case report of 3 or fewer patients. Our report does not contain any identifying information and is reviewing the presentation of one patient, therefore IRB approval was not requested.

- Consent for publication

- The patient provided written informed consent for publication of the case report. It is included here.

- Availability of data and materials

- See references above

- Competing interests

- The authors declare that they have no competing interests.

- Funding 
- Request waiver as submitting as a medical resident without access to robust funding.

- Authors' contributions

- SPB: Wrote manuscript

- MN: Reviewed manuscript and provided detailed feedback and support

- Acknowledgements

- We thank the patient and her family for allowing us to care for her and share this information to allow others to better care for patients in the future.

- Authors' information (optional)

- SPB: Current PGY2 Internal Medicine Resident at UAB with career aspirations in Hematology/Oncology

- MN: Assistant Professor in the Division of Hematology/Oncology

\section{References}

1. Shenoy PJ, Malik N, Nooka A, et al. Racial differences in the presentation and outcomes of diffuse large B-cell lymphoma in the United States. Cancer. 2011;117(11):2530-2540. doi:10.1002/cncr.25765

2. Møller MB, Pedersen NT, Christensen BE. Diffuse large B-cell lymphoma: clinical implications of extranodal versus nodal presentation-a population-based study of 1575 cases. British Journal of Haematology. 2004;124(2):151-159. doi:10.1046/j.1365-2141.2003.04749.x

3. Armitage JO, Weisenburger DD. New approach to classifying non-Hodgkin's lymphomas: clinical features of the major histologic subtypes. Non-Hodgkin's Lymphoma Classification Project. Journal of Clinical Oncology: Official Journal of the American Society of Clinical Oncology. 1998;16(8):2780-2795. doi:10.1200/JC0.1998.16.8.2780

4. Bernstein SH, Unger JM, Leblanc M, Friedberg J, Miller TP, Fisher RI. Natural history of CNS relapse in patients with aggressive non-Hodgkin's lymphoma: a 20-year follow-up analysis of SWOG 8516 -- the Southwest Oncology Group. J Clin Oncol. 2009;27(1):114-119. doi:10.1200/JC0.2008.16.8021

5. Mead GM, Kennedy P, Smith JL, et al. Involvement of the central nervous system by non-Hodgkin's lymphoma in adults. A review of 36 cases. Q J Med. 1986;60(231):699-714.

6. Morrison SF, Nakamura K. Central Mechanisms for Thermoregulation. Annu Rev Physiol. 2019;81:285-308. doi:10.1146/annurev-physiol-020518-114546

7. Seymour JF, Talpaz M, Cabanillas F, Wetzler M, Kurzrock R. Serum interleukin-6 levels correlate with prognosis in diffuse large-cell lymphoma. J Clin Oncol. 1995;13(3):575-582. doi:10.1200/JC0.1995.13.3.575

8. Landman RE, Wardlaw SL, McConnell RJ, Khandji AG, Bruce JN, Freda PU. Pituitary Lymphoma Presenting as Fever of Unknown Origin*. The Journal of Clinical Endocrinology \& Metabolism. 2001;86(4):1470-1476. doi:10.1210/jcem.86.4.7389 
9. Salih SB, Saeed AB, Alzahrani M, Al Qahtani M, Haider A, Palker V. Primary CNS lymphoma presenting as fever of unknown origin. Journal of Neuro-Oncology. 2009;93(3):401-404. doi:10.1007/s11060-008-9777-0

10. Geng $M$, Song $Y$, Xiao $H$, et al. Clinical significance of interleukin-10 concentration in the cerebrospinal fluid of patients with primary central nervous system lymphoma. Oncology Letters. 2020;21(1):1-1. doi:10.3892/ol.2020.12263

11. Petersdorg RG, Beeson PB. Fever of unexplained origin: report on 100 cases. Medicine (Baltimore). 1961;40:1-30. doi:10.1097/00005792-196102000-00001

12. Sehn LH, Martelli M, Trněný $M$, et al. A randomized, open-label, Phase III study of obinutuzumab or rituximab plus CHOP in patients with previously untreated diffuse large B-Cell lymphoma: final analysis of GOYA. Journal of Hematology \& Oncology. 2020;13(1). doi:10.1186/s13045-020-00900-7

13. Wilson MR, Eyre TA, Martinez-Calle N, et al. Timing of high-dose methotrexate CNS prophylaxis in DLBCL: an analysis of toxicity and impact on R-CHOP delivery. Blood Advances. 2020;4(15):35863593. doi:10.1182/bloodadvances.2020002421

\section{Figures}

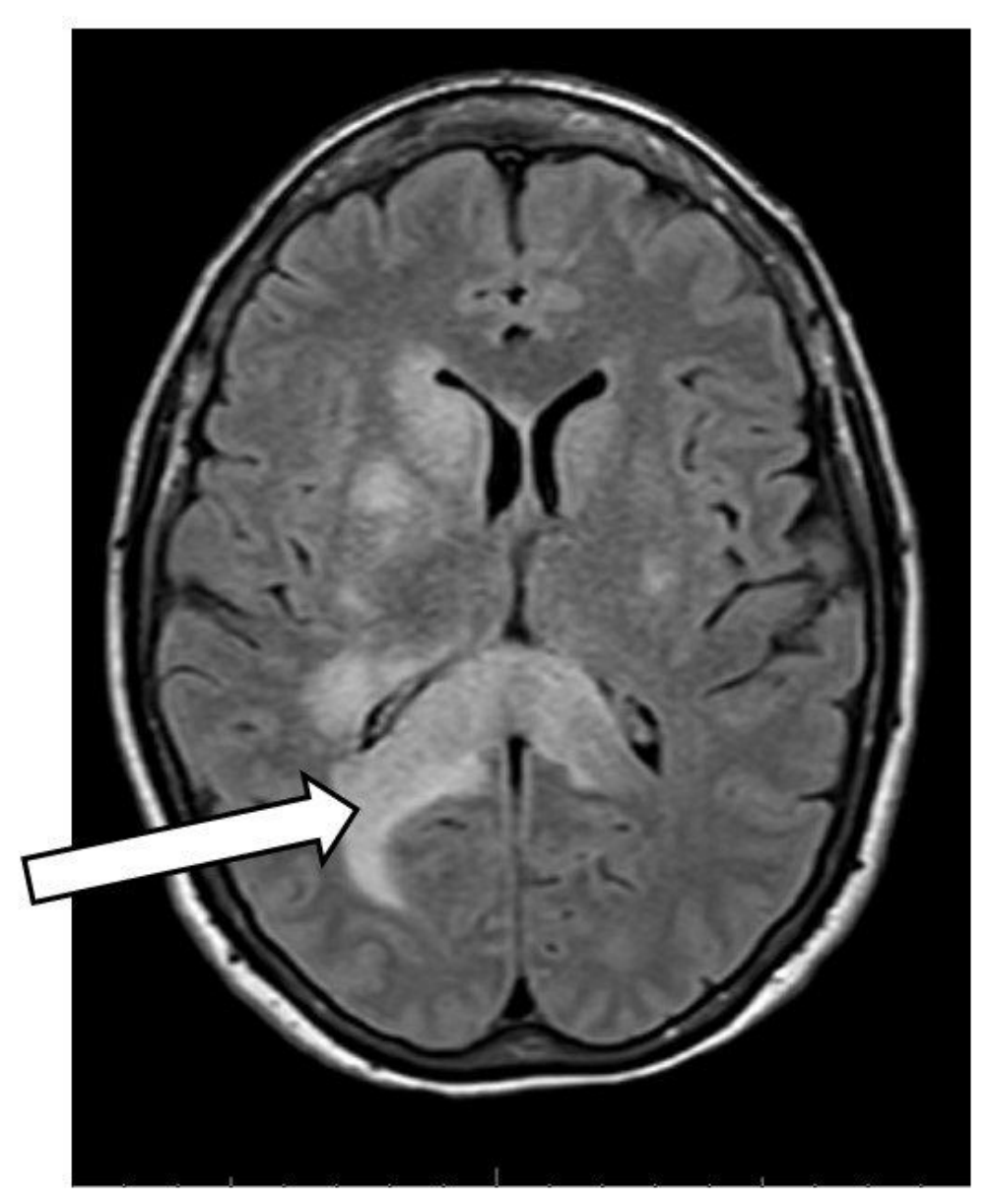

Figure 1 
Diffuse parenchymal lymphomatosis involvement on MRI Brain Axial FLAIR View 